\title{
SIMULATING THE OPERATIONAL CONTROL OF FREE RANGING AGVS
}

\author{
Mark B. Duinkerken \\ Tiemen ter Hoeven \\ Gabriel Lodewijks \\ Faculty of Mechanical, Maritime and Materials Engineering \\ Delft University of Technology \\ Mekelweg 2 \\ 2628 CD Delft, THE NETHERLANDS
}

\begin{abstract}
Most transport systems using automated guided vehicles (AGVs) are centrally controlled and use fixed, pre-defined routes. Incidents cannot be handled as part of the common routine. Instead of using fixed path layouts, new trajectory planners for AGVs are developed that utilize the complete available space. To accommodate the increased flexibility, new operational controllers must be able to adapt to small deviations and incidents. In this paper an operational controller is presented that aims to satisfy two conflicting goals. First, the controller directs an AGV along a preplanned trajectory with high accuracy. Second, the controller will avoid conflicts with static and dynamic obstacles. These conflicts are caused by small deviations between planned and realized paths, as well as by incidents like equipment failure. A simulation model is built to study the performance of this controller. The quality is compared to a PI-controller without collision avoidance characteristics.
\end{abstract}

\section{INTRODUCTION}

In current AGV systems, the automatic guided vehicles move through the facility along predetermined paths (van Dam et. al. 2004). However, with the increasing computing power available for onboard use, it becomes possible to determine AGV trajectories more frequently. Instead of using a fixed path layout, the onboard computer of an AGV can calculate a trajectory each time a transportation job is issued and even recalculate a trajectory during the execution of the transport job. These paths can be established, taking into account the actual positions of both static (infrastructural) obstacles and dynamic obstacles (like other driving AGVs). The expected advantages of this approach over the traditional design are dynamic obstacle avoidance, more flexibility, more efficient use of infrastructure and higher accuracy in the expected time of arrival. These characteris- tics will eventually lead to AGV systems with better utilization, more flexibility and high punctuality.

\subsection{Free range routing}

To use the free ranging and positioning capacities of $\mathrm{AGVs}$, a controller is required to calculate a path (trajectory) each time an $\mathrm{AGV}$ is assigned to a transportation job. Such a controller can take into account the due time of the transportation job, the location of obstacles on the infrastructure and the expected traffic density. The controller will consist of a tactical and an operational part. On the tactical level, a path is calculated in combination with a speed profile (Barraquand and Latombe 1991). This will guarantee a timely arrival at the destination location, assuming that the AGV is capable of realizing the speed profile on the given path. On the operational level, a controller compares the expected position with the actual position of the AGV and determines control signals for steering and propulsion. The operational controller must also allow the AGV to deviate from the planned path in case an (unforeseen) obstacle or another AGV is encountered (Khatib 1986). Of course, sometimes a new calculation on the tactical level can be required.

A new control model called DEFT (Dynamic Evasive Free-ranging Trajectories) is developed that allows for dynamic free range routing of AGVs. As a starting point, a microscopic pedestrian model called NOMAD (Hoogendoorn and Bovy 2002a, Hoogendoorn and Bovy 2002b) is used, which was developed to describe the behaviour of pedestrians, choosing their paths within the infrastructure. For DEFT, this model is modified and extended to cope with the control of AGVs. It needs to be determined whether or not an AGV system with this type of control outperforms the more traditional type of control. Because of the layered structure of the DEFT model, it is possible to simulate the strategic, tactical and operational levels sepa- 


\section{Duinkerken, ter Hoeven, and Lodewijks}

rately. More information on the merits and development of DEFT and can be found in (Duinkerken 2006).

\subsection{Research goal}

The focus of this paper is on the operational control of free ranging AGVs. A logical first step is to set up a simple simulation with fixed driving paths to study how the operational level of the model copes with AGV encounters. This paper discusses the design and test of such an operation controller.

The objective of the DEFT operational controller is twofold:

- Follow a path as accurately as possible, when no obstacles are found

- Deviate from the path when an obstacle or AGV is encountered.

The main research questions are:

- What path-following accuracy can be reached using the DEFT-controller, compared to an existing PI-controller?

- What values should be given to the weighting factors and parameters of the optimal control law equation to obtain good path-following and object evasion behaviour?

The remainder of this paper is organized as follows. In the next chapter, the trajectory control model is defined and some information is given on the PI-controller which is used for the comparison with the DEFT operational controller. Chapter 3 presents the structure and mathematical background of the DEFT model. In chapter 4 the simulation model is described. In chapter 5 the experiments and results are given and the final chapters contains the conclusions and recommendations for further work.

\section{DESCRIPTION OF AGV OPERATIONAL CONTROL}

\subsection{Trajectory tracking control}

It is assumed that a trajectory from the current position of the AGV to a desired destination is planned on a higher level (the tactical level). The AGV needs to be able to follow this trajectory that defines the desired position as a function of time. The trajectory does not only define the string of coordinates the centre of the vehicle has to follow, but also the angle between the AGVs centreline and the direction of the path, know as the crabbing angle, to gain an increased manoeuvring capability.

To correct any deviations from the desired trajectory the vehicles require a controller that adjusts the steering angles and speed of the wheels. The task of this controller can be split up into two parts. The first part involves the minimization of the lateral error by controlling the steering angles, and the second part consists of the minimization of the longitudinal error by controlling the speed of the vehicle. Figure 1 shows how these errors are defined.

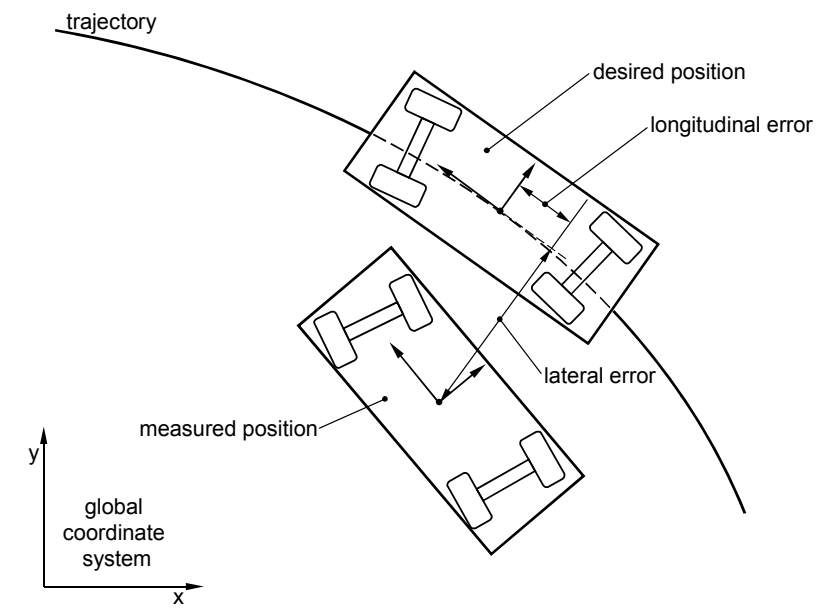

Figure 1: Errors that Occur when the Measured Position Deviates from the Desired Position

Due to the relatively low operating speeds wheel slip resulting from side forces is not considered. This entails that there will be no velocity component perpendicular to the main axis of the wheels. Furthermore, in the absence of wheel slip the movement of the vehicle can be determined by kinematic relations.

\subsection{The PI controller}

The performance of the DEFT controller presented in this paper will be compared to a previously developed motion controller (Duinkerken, Nuttall and Lodewijks 2005). This proportional-integral controller, called PI-controller in this paper, has no collision avoidance characteristics. It is expected that it outperforms the DEFT controller in situations without obstacles (because a PI-controller will be better than the proportional behaviour of the DEFT controller), but of course it will fail to avoid collisions.

Minimization of the vehicle's lateral error will be accomplished by letting each wheel individually steer towards its desired position. If both wheels reach their projected positions, which can be calculated from the current position on the trajectory and the desired crabbing angle, the lateral error of the vehicle's centre is reduced to zero. In effect each wheel will get its own proportional controller that adjusts the steering angle to minimize the lateral distance between the projected and actual wheel position.

As the AGV adjusts its steering angles to stay on its predefined trajectory, the actual travelled distance will start to vary from the expected travelled distance. This differ- 


\section{Duinkerken, ter Hoeven, and Lodewijks}

ence results in a growing longitudinal error. To correct this error the AGV will need to make speed corrections. Furthermore, to be able to maintain the desired speed the motor's power supply has to compensate for varying mechanical resistances resulting from changing running surfaces ranging from smooth to very rough floors. A model of the vehicle's driveline is created (Stadler 1995) and a PIcontroller was selected to control this system. A more detailed description of the model can be found in (Duinkerken et. al. 2005).

\section{DEFT OPERATIONAL CONTROLLER}

The goal of DEFT operational controller is to let the AGV follow the defined trajectory as closely as possible. To ensure that this goal is pursued, a cost-minimizing approach can be used (Hoogendoorn and Bovy 2003). Deviation from the original (ideal) velocity path comes at a cost. An AGV can alter its velocity by accelerating or decelerating with a certain magnitude and direction. This can be considered as a control vector. Applying acceleration comes at a cost, and so does proximity to other AGVs or obstacles. The acceleration that is 'cheapest' when these costs are added will be applied, and is called the optimal control vector. Figure 2 shows the structure of the operational level with its inputs and output. The velocity and position of other AGVs in the same system are dynamic inputs of the model, which should be updated at a (very short) interval.

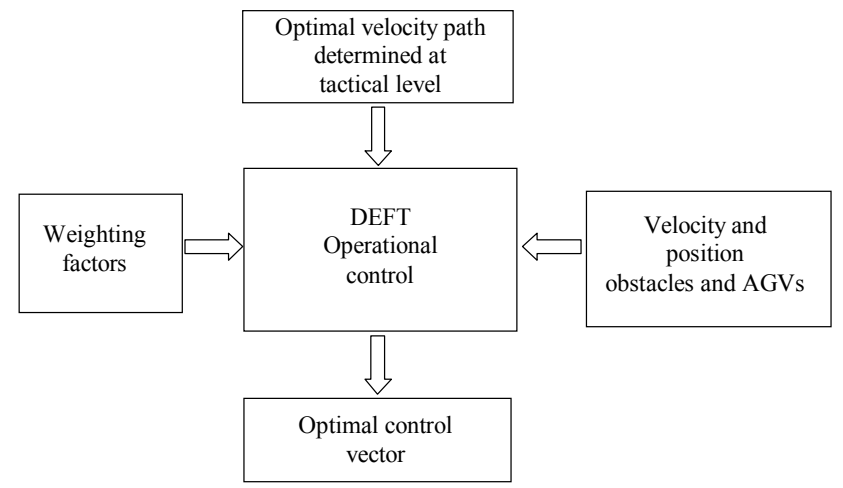

Figure 2: Structure of the Operational Level

The control action of the operational level is based on four types of running costs: costs assigned for deviating from the ideal velocity vector, costs assigned for deviating from the reference position, costs assigned for proximity to other AGVs or obstacles and costs assigned for accelerations. For each time $t$, the combined costs will be minimized by the optimal acceleration law, resulting in a control action.

\subsection{Running cost factor 1: velocity error costs}

The first running cost factor assigns a cost to the difference between the planned and realized velocity. The velocity vector will have to be derived from the prescribed path. The prescribed velocity vector is always in the direction of the path. In case the $\mathrm{AGV}$ is on its prescribed path, the minimal costs will always be in the correct direction.

\subsection{Running cost factor 2: position error costs}

When a vehicle wanders of its path, it will not be steered back to the path. The second cost factor is used to assign costs to the error in position. The input of the running cost factor for position error is a position reference vector composed from the lateral and longitudinal difference in position with the prescribed path point. The simplest way of defining this vector is by simply calculating the difference in position between the current and path position at time $t$. This means that if an AGV follows this reference vector, it will always try to minimize the distance between itself and the reference point at a given time.

As long as the error between the reference position and the current position is kept small, the method described above poses no problems, and the AGV will follow its path quite accurately. However, when we are dealing with larger errors in position, caused by for example interaction with obstacles or other AGVs, simply taking the shortest route to the reference position point may cause problems. To improve the behaviour of the controller for situations with larger longitudinal deviations, the position reference vector can also be redefined: the lateral error is the lateral difference between the current position and the nearest position on the path. The longitudinal error consists of the difference in path length between the reference position and the current position. The longitudinal error vector is always defined tangent to the path at the nearest path-point. In this way, the AGV will try to minimize the distance between its current position and the reference position without cutting corners.

Both path-following methods will be available in the experimental version of the operational controller. A parameter is introduced that applies to both methods of reference: the balance between longitudinal and lateral cost of deviation from the path. The value of this balance will influence the way $\mathrm{AGVs}$ deviate from their path when evasive actions are necessary.

\subsection{Running cost factor 3: costs of proximity to objects}

This running cost factor describes the cost of the proximity to other vehicles or obstacles. For AGV application, it is important that the size of the vehicles themselves is used to calculate the smallest distance to an object. Also, when an AGV encounters an object, we would like it to take evasive action in efficient (shortest) direction. This means that the centre of objects should also be taken into account, to determine the shortest way around an object. According to the optimization theory used in the model, the only 
mathematical demand for a proximity function is that it should be a time-independent function of the position and speed relative to other vehicles and obstacles.

\subsection{Running cost factor 4: acceleration costs}

The last running cost factor assigns a cost to acceleration, and sets the balance between longitudinal and lateral acceleration. The definition of the cost of acceleration should limit the resulting accelerations to suit the physical possibilities of AGVs. These limits should be applied to the outcome of the optimal acceleration law, which will be defined later.

\subsection{Definition of the optimal acceleration law}

Now that the running cost factors have been defined, we can derive the optimal control vector. First, each cost factor $\mathrm{k}$ is multiplied by a weighting coefficient $\mathrm{c}_{\mathrm{k}}$. To find the optimal acceleration, we apply the Maximum principle of Pontryagin. Details can be found in (Hoogendoorn and Bovy 2003). For application to AGVs, the acceleration vector must be translated into steering angles and velocity.

\section{SIMULATION AND EMULATION}

\subsection{Model description}

To test the performance of the AGV controller, an emulation model is used to replace the actual hardware that the control logic will interface with. The models interface is set up in the same form as if it were connected to the real system. Because emulation only replaces part of the real system with a model, emulation has more credibility than simulation when it comes to using the model to predict the performance of real systems. The controller is coded into an AGV control program using the Delphi programming package.

A discrete event simulation model of the real world AGV was built using Delphi in combination with the TOMAS simulation package (Veeke and Ottjes 2002). Both the controller for the AGV and the emulator of the AGV use a fixed discrete time step. The resulting program defines several moves of the AGV on different kinds of paths it has to follow, includes the dynamics associated with the servos and the motors of the AGV and also models the observation system for position determining. In this program, the task of the emulation model is to process the signal coming from the controller with the associated servo and motor dynamics to determine the new state of position and velocity. The emulation study conducted with this program enables testing performance of the real AGV running on a defined trajectory with the developed control system.

\subsection{Input parameters}

The performance of the controller depends on some circumstantial factors and parameters:

- Trajectories of AGVs in simulation

- Definition of obstacles

- Weighting factors $\mathrm{c}_{1}, \mathrm{c}_{2}, \mathrm{c}_{3}$ and $\mathrm{c}_{4}$ of the running costs

- Object distance scaling factor

- Balance factor for longitudinal / lateral position error costs

- Balance factor for longitudinal / lateral acceleration costs

- Time interval used by approximation method

\subsection{Performance indicators}

The performance of the operational controller will be evaluated using these performance indicators, separated for the two objectives:

For objective 1, the trajectory following quality:

- Lateral and longitudinal deviation from the path

- Angular deviation from the prescribed driving angle

- Overshoot, $5 \%$ settling time and damping of the controller.

For objective 2, the collision avoidance quality:

- Range of close encounters with other AGVs and obstacles

- Deviation from the planned trajectory.

The deviations mentioned above are evaluated with the same frequency the AGV controller uses. To obtain the performance over a trajectory, the mean square error is calculated.

\section{EXPERIMENTS AND RESULTS}

A number of experiments were conducted to evaluate the performance for both research objectives. Three tests were done to derive the controller's characteristics on pathfollowing and to tune it for accuracy. Two more tests show the behaviour of the AGV when respectively evading obstacles and vehicles. The final test will use the optimal settings found for obstacle evasion to evaluate the remaining path-following accuracy. 


\subsection{Lane Change Test}

The lane change test uses only a lateral error as an input signal; this means that only the steering of the AGV is controlled, and that its speed is fixed at the target velocity. A straight path with a lateral discontinuity of $0.10 \mathrm{~m}$ is defined. This path acts as a step function on the input of the lateral controller, and can be used to derive the controller's characteristics. We will measure the lateral deviation from the path of the AGV, and tune the controller's settings for accuracy. Optimizing for all three performance indicators, the adequately tuned settings are found at $\mathrm{C}_{1}=10, \mathrm{C}_{3}=2$ and $\mathrm{C}_{2}=100$. Table 1 compares the results to the results of the PI controller.

Table 1: Results of Lane Change Test

\begin{tabular}{|l|c|c|c|}
\hline & $\begin{array}{c}\text { overshoot } \\
(\%)\end{array}$ & $\begin{array}{c}5 \% \text { settling } \\
\text { time (s) }\end{array}$ & damping \\
\hline PI controller & 4.33 & 0.42 & 0.71 \\
\hline DEFT & 0.75 & 0.42 & 0.69 \\
\hline
\end{tabular}

From the comparison, it can be concluded that the controllers are not very different in performance, which is not entirely surprising, since the PI lateral controller is also a proportional second-order feedback system. The most significant difference is that of the overshoots; the DEFT controller has an overshoot of less than a fifth of that of the PI controller. In practice however, both overshoots are sufficiently small to be used in AGV control.

\subsection{Straight path test}

To test the longitudinal controller, a step speed signal is used as input. From velocity zero, the AGV will have to accelerate to $1.0 \mathrm{~m} / \mathrm{s}$, which is the target speed, whilst minimizing the longitudinal position error. This way an insight in the longitudinal positioning accuracy of the controller is gained. The longitudinal deviation from the path will be measured, and the controller will be tuned for longitudinal accuracy. The test results for these settings are presented in Table 2.

Table 2: Results of Straight Path Test

\begin{tabular}{|l|c|c|c|c|}
\hline & $\begin{array}{c}\text { overshoot } \\
(\%)\end{array}$ & $\begin{array}{c}5 \% \\
\text { settling } \\
\text { time (s) }\end{array}$ & damping & $\begin{array}{c}\text { steady } \\
\text { state } \\
\text { error } \\
(\mathrm{m})\end{array}$ \\
\hline PI controller & N.A. & N.A. & N.A. & 0.00 \\
\hline DEFT & 8.59 & 0.13 & 0.70 & 0.02 \\
\hline
\end{tabular}

For the PI-controller, detailed information on the results of the straight path test was not available. However, it was shown that the steady state (position) error is almost equal to zero, which can be expected of the PI-controller. The DEFT controller has a steady state error of about 2 centimetres, which is equal to $4 \%$ of the vehicle's length. The
PI controller has both a considerably longer settling time and larger position error at the start of the run.

\subsection{Realistic trajectory}

The final test to compare the controllers is a combined test of both the lateral and longitudinal controllers. Using a 'realistic' trajectory the longitudinal, lateral and orientation mean square errors of the AGV will be compared. The settings found from the first two tests will be used to achieve the best results in path-following accuracy. The corresponding test results are compared to the results of the PI controller for the same trajectory in Table 3.

Table 3: Results of Combined Test (Mean Square Errors)

\begin{tabular}{|l|l|l|l|}
\hline & $\begin{array}{l}\text { lateral } \\
\left(10^{-3} \mathrm{~m}^{2}\right)\end{array}$ & $\begin{array}{l}\text { longitudinal } \\
\left(10^{-3} \mathrm{~m}^{2}\right)\end{array}$ & $\begin{array}{l}\text { orientation } \\
\left(10^{-3} \mathrm{~m}^{2}\right)\end{array}$ \\
\hline PI controller & 0.01 & 0.25 & 3.34 \\
\hline DEFT & 0.40 & 0.29 & 0.02 \\
\hline
\end{tabular}

From the comparison, it appears that the PI controller is slightly more accurate for the combined test. The lateral accuracy is significantly better than that of the DEFT controller. The longitudinal accuracy is almost equal, but the orientation accuracy of the DEFT controller is significantly better. Again, the performance of both controllers seems sufficient for AGV control purposes.

\subsection{Obstacle Evasion}

To test the obstacle evasion behaviour of the controller, and to tune it for the best results, a path will be defined which runs straight through an obstacle. The controller's parameters will be varied to obtain satisfactory evasion behaviour. For the evasion tests, a nominal velocity value of 0.25 metres per second was chosen because this is a much more realistic value for the mini-AGV than the $1.0 \mathrm{~m} / \mathrm{s}$ used for the accuracy tests. Multiplied by the scale factor of 25 , this means that a real-sized AGV would drive at 6.25 metres per second, which is considered rather high.

During the experiments it was obvious that by adjusting the acceleration / steering balance different types of behaviour can be generated. Ideally, the AGV would drive past obstacles at a reasonable distance, and return to its path as soon as possible. If the object cost slope factor is set too high, the AGV drives to close to the obstacle, and because of the very steep cost function, every steering action causes a considerable rise or decline of the object costs. This causes unstable behaviour. On the other hand, if the distance threshold is too low, the AGV is suddenly confronted with the already very high costs of the obstacle in front of it, which again causes unstable behaviour. Care must thus be taken while setting the obstacle cost parameters. After tuning the parameters, the result of the obstacle evasion test was obtained as shown in Table 4. 
Table 4: Results of Obstacle Evasion Test

\begin{tabular}{|l|l|l|}
\hline & $\begin{array}{l}\text { minimum } \\
\text { distance } \\
(\mathrm{m})\end{array}$ & $\begin{array}{l}\text { maximum } \\
\text { longitudinal } \\
\text { deviation } \\
(\mathrm{m})\end{array}$ \\
\hline DEFT & 0.32 & 0.65 \\
\hline
\end{tabular}

The AGV has a stable driving path past the obstacle, whilst returning quickly to the reference position (see Figure 4). The minimum distance between the AGVs safety-ellipse and the obstacle is 0.32 metres, and the maximum longitudinal deviation (distance behind) is $0.65 \mathrm{~m}$.

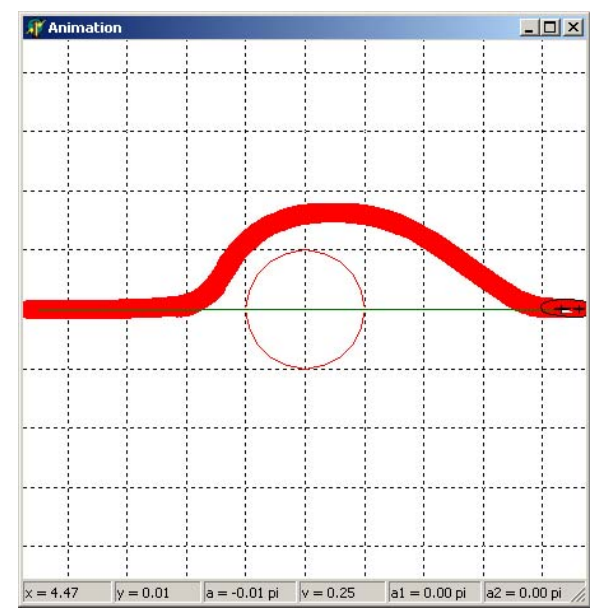

Figure 4: Resulting Obstacle Evasion Path (Trajectory from Left to Right)

\subsection{Vehicle evasion}

The vehicle evasive behaviour was tested by defining to straight paths, at a $45^{\circ}$ angle, each path driven by an AGV. One of the AGVs had a head-start of 0.50 metres, which should ensure that the other AGV gives way. The two AGVs did not collide; the minimum distance reached between the vehicles was $0.22 \mathrm{~m}$, as the test results indicate. By tuning the longitudinal error costs and the longitudinal / lateral error cost balance, even more satisfactory results were obtained.

\subsection{Remaining accuracy}

The DEFT controller is not expected to have the same positioning accuracy with the settings derived from the evasion tests. To optimize the evasive characteristics, the weight coefficients for the four cost components are modified and not equal to the settings found for optimal path following. The remaining accuracy is tested by submitting the controller to the same realistic trajectory test that was done earlier. The settings found from the evasion tests can be considered a good compromise for all the situations tested. Since the longitudinal error costs were lowered, we can expect a larger longitudinal steady state error. In general the AGV will be behind on its path but given time, the distance behind will be minimized to the steady state error. To indicate the kind of accuracy that can be expected from these settings, the combined accuracy test was done again, and is compared to the results of the PI controller; see Table 5 .

Table 5: Remaining Accuracy using Evasive Settings (Mean Square Errors)

\begin{tabular}{|l|c|c|c|}
\hline & $\begin{array}{c}\text { lateral } \\
\left(10^{-3} \mathrm{~m}^{2}\right)\end{array}$ & $\begin{array}{c}\text { longitudinal } \\
\left(10^{-3} \mathrm{~m}^{2}\right)\end{array}$ & $\begin{array}{c}\text { orientation } \\
\left(10^{-3} \mathrm{~m}^{2}\right)\end{array}$ \\
\hline PI controller & 0.01 & 0.25 & 3.34 \\
\hline DEFT & 0.40 & 0.29 & 0.02 \\
\hline DEFT evasive & 0.15 & 31.68 & 2.23 \\
\hline
\end{tabular}

Surprisingly, the lateral accuracy has improved for the evasive settings. This is caused by the trailing in of the AGV in the bends, when it is longitudinally behind. There is a significant decrease in longitudinal and orientation accuracy for the evasive settings. The average distance behind (not shown in the table, but indicated by the high longitudinal mean square error) is $0.18 \mathrm{~m}$, which is not very accurate. However, these more passive settings are needed to ensure proper obstacle and vehicle evasion.

\section{CONCLUSIONS}

The implementation of the DEFT operational controller for AGVs has been done by adding AGV dimensions and limited freedom of motion to a pedestrian control model. These basic adaptations have been successful; the controller is able to control the direction and speed of the AGVs. By adding an extra running cost for position deviation and redefining the optimal control law, it is now possible to follow a pre-defined two-dimensional path in time.

A new proximity function was drawn up, which uses both the centre of an object and the absolute distance to the object to create predictable and efficient object evasion. This definition is directly used for obstacle evasion. For vehicle evasion, a very simple traffic decision model has been drawn up to improve the efficiency of vehicle evasion. This model determines in which direction the AGVs will take evasive actions, and if they will slow down for other vehicles.

Tests proved that when tuned for accuracy, the DEFT controller can achieve the same order of accuracy as a PI controller. The DEFT controller performs slightly better on the lane change test. Due to the fact that it is solely a proportional controller, a longitudinal steady state error will always occur on the straight path test. The reference longitudinal controller is a proportional integral controller, and does not have this problem. However, this PI controller has a considerably longer settling time on the straight path test.

The most interesting aspect of the DEFT model is its ability to take evasive action when an object is encoun- 


\section{Duinkerken, ter Hoeven, and Lodewijks}

tered. Using a simple obstacle evasion test and testing encounters with other AGVs at several angles, a set of evasive settings was determined, for which most situations are dealt with adequately. Obstacle evasion is very reliable and predictable, and proves that using DEFT for operational control of AGVs has certainly been worth the effort. Obstacles of all shapes and sizes can be avoided efficiently by the controller, as long as the path to be followed is relatively simple. Vehicle evasion was less successful than obstacle evasion. The relatively simple traffic decision model sometimes causes inefficient evasive actions when a vehicle and obstacle are encountered simultaneously, for example. The vehicle evasion tests done do show that traffic can be regulated by simply indicating which vehicle should give way, and whether it should pass in front or behind. The controller is then flexible enough to ensure a safe distance between the vehicles.

Summarizing, the performance results of the DEFT operational controller are very promising. Using the evasive settings, the path-following accuracy may not be as good as that of a PI controller, but this is compensated by its flexibility. Obstacle evasion has proven to be very reliable; the vehicle evasion behaviour still can be improved by applying a better traffic decision scheme.

\section{FURTHER DEVELOPMENTS}

Many questions remain on the functioning of the controller and the behaviour of the AGVs. Some recommendations are given here for further study.

A running cost could be added for deviation from the prescribed driving angle. When the front and rear steering angle are decoupled, it may be possible to follow a path containing sections of crabbing motion. Since the current model has only an acceleration vector as its output, some additions will have to be done to obtain separate acceleration and orientation angles for the front and rear axles of an AGV.

More research should be done on time step dependent stability. The time step of the approximation method used to calculate the optimal acceleration could be decoupled from the position, velocity and distance information update time step. It may then be possible to maintain stable AGV behaviour when the information fed to the AGV has a lower update frequency.

The behaviour of the controller when it encounters obstacles or vehicles should be studied further. The cause of the steering and velocity fluctuations will have to be investigated, for example by keeping track of all running costs during a test run, and comparing these with the behaviour of the AGV. More complex obstacles could be defined, to test the evasive behaviour for concave shapes, for example.

The goal of the research project is improving the capacity and accuracy of an automated transport system. For this, the DEFT model consists of a strategic, tactical and operational layer. The combination of the tactical controller with the operational controller has yet to be tested. After that, the controllers can be integrated with a strategic controller. A laboratory using the complete DEFT model for the study of a scale version of an automated container terminal is under development.

\section{ACKNOWLEDGMENTS}

This work was supported in part by the Next Generation Infrastructures Foundation.

\section{REFERENCES}

Barraquand, J. and J.C. Latombe. 1991. Nonholonomic multibody mobile robots: Controllability and motion planning in the presence of obstacles. In IEEE International Conference on Robotics \& Automation, 23282335.

Dam, K.H. van, J.A. Ottjes, G. Lodewijks, Z. VerwaterLukszo and R.W. Wagenaar. 2004. Intelligent infrastructures: distributed intelligence in transport system control - an illustrative example. In: Proceedings of the International Conference on Systems, Man and Cybernetics. IEEE, The Hague, 4650 - 4654.

Duinkerken, M.B., A.J.G. Nuttall, J.A. Ottjes and G. Lodewijks. 2005. Developing an AGV motion controller using simulation, emulation and prototyping. In: IEEE Intelligent Vehicles Symposium Proceedings. Las Vegas NV, USA.

Duinkerken, M.B., M. van der Zee and G. Lodewijks. 2006. Dynamic free range routing for automated guided vehicles. In: IEEE International Conference on Networking, Sensing and Control. Ft. Lauderdale.

Hoogendoorn, S.P. and P.H.L. Bovy. 2002a. Pedestrian route-choice and activity scheduling theory and methods. In: Transportation Research B. Vol. 38, No. 2, 169-190.

Hoogendoorn, S.P. and P.H.L. Bovy. 2002b. Normative pedestrian behaviour theory and modelling. In: Michael A.P. Taylor (Ed.), Transportation and traffic theory in the 21st century. Oxford: Pergamon, Elsevier science, 219-245.

Hoogendoorn, S.P. 2003. Pedestrian travel behavior modeling. In: Proceedings of the 10th international Conference on Travel Behavior Research, Lucerne.

Khatib, O. 1986. Real-time obstacle avoidance for manipulators and mobile robots. In: The International Journal of Robotics Research. 90-98.

Stadler, W. 1995. Analytical robotics and mechatronics. New York, McGraw-Hill.

Veeke, H.P.M. and J.A. Ottjes. 2002. TOMAS: Tool for object-oriented modelling and simulation. In: Proceedings of the Business and Industry Simulation Symposium. Washington D.C.. 


\section{AUTHOR BIOGRAPHIES}

MARK B. DUINKERKEN obtained his Master degree in Applied Mathematics at the Delft University of Technology (1991). He is currently an Assistant Professor in Logistic Engineering at the faculty of Mechanical, Maritime and Materials Engineering of Delft University of Technology. His specialization is modeling and simulation of logistic processes and optimizing the planning and control of logistic processes with OR-techniques. He participates in several public-private research projects aimed at the design of high capacity container terminals. Other work includes research concerning City Logistics, the development of an AGV-laboratory, student courses in computer simulation and the development of simulation tools. His e-mail address is $<$ m.b. duinkerken@wbmt. tudelft.nl>.
TIEMEN TER HOEVEN studied mechanical engineering at Delft University of Technology, The Netherlands, from which he obtained a Master degree in 2005. He specialized in transport and logistic engineering.

GABRIEL LODEWIJKS studied mechanical engineering at Twente University and Delft University of Technology, The Netherlands, from which he obtained a Master degree (cum laude) in 1992. He specialized in transport technology, material engineering and dynamics, and obtained his Ph.D. degree at Delft University of Technology on the dynamics of belt systems in 1996. In November 2000 he was appointed Professor of Transport Technology and Logistic Technology at the faculty of Mechanical, Maritime and Materials Engineering of Delft University of Technology. His main interest is in belt conveyor technology, DEM applications in transport technology, automation of transport systems, material engineering and dynamics. 